\title{
S100 family signaling network and related proteins in pancreatic cancer (Review)
}

\author{
YI-FEI JI $^{1 *}$, HUA HUANG ${ }^{2 *}$, FENG JIANG ${ }^{1}$, RUN-ZHOU NI $^{1}$ and MING-BING XIAO ${ }^{1}$ \\ Departments of ${ }^{1}$ Gastroenterology and ${ }^{2}$ Pathology, Affiliated Hospital of \\ Nantong University, Nantong, Jiangsu 226001, P.R. China
}

Received September 18, 2013; Accepted January 20, 2014

DOI: $10.3892 /$ ijmm.2014.1633

\begin{abstract}
The occurrence and development of pancreatic cancer is a complex process convoluted by multi-pathogenies, multi-stages and multi-factors. S100 proteins are members of the S100 family that regulate multiple cellular pathways related to pancreatic cancer progression and metastasis. S100 proteins have a broad range of intracellular and extracellular functions, including the regulation of protein phosphorylation and enzyme activity, calcium homeostasis and the regulation of cytoskeletal components and transcriptional factors. S100 proteins interact with receptor for advanced glycation end-products (RAGE), p53 and $\mathrm{p} 21$, which play a role in the degradation of the extracellular matrix (ECM) and metastasis, and also interact with cytoskeletal proteins and the plasma membrane in pancreatic cancer progression and metastasis. S100A11 and S100P are significant tumor markers for pancreatic cancer and unfavorable predictors for the prognosis of patients who have undergone surgical resection. Recently, S100A2 has been suggested to be a negative prognostic biomarker in pancreatic cancer, and the expression of S100A6 may be an independent prognostic impact factor. The expression of S100A4 and S100P is associated with drug resistance, differentiation, metastasis and clinical outcome. This review summarizes the role and significance of the S100 family signaling network and related proteins in pancreatic cancer.
\end{abstract}

\section{Contents}

1. Introduction

2. The S100 family

3. S100 proteins as molecular targets in pancreatic cancer

Correspondence to: Professor Ming-Bing Xiao, Department of Gastroenterology, Affiliated Hospital of Nantong University, 20 Xi Si Road, Nantong, Jiangsu 226001, P.R. China

E-mail:xmb73@163.com

*Contributed equally

Key words: S100 proteins, pancreatic cancer, expression, biomarkers, molecular targets
4. S100 protein expression in pancreatic cancer

5. Conclusion

\section{Introduction}

The prevention and treatment of pancreatic cancer is a difficult issue worldwide, as pancreatic cancer is associated with higher malignancy and metastatic rates from the early stage of disease (1). More than $95 \%$ of patients diagnosed with pancreatic cancer succumb to the disease and half of these patients within six months after diagnosis $(2,3)$. Radio- and chemotherapy do not have major effects on the survival of pancreatic cancer patients $(2,4)$; thus, surgery remains the optimal treatment method. Prognosis mainly depends on early diagnosis and treatment. Therefore, it is of great importance to identify novel diagnostic markers and to explore related proteins involved in signaling pathways associated with the occurence, development and metastasis of pancreatic cancer. S100 proteins interact with multiple molecular targets in both a calcium-dependent and -independent manner $(5,6)$. They regulate multiple cellular pathways that play key roles in pancreatic cancer progression and metastasis $(7,8)$. S100 proteins may thus be early diagnostic biomarkers.

\section{The S100 family}

The S100 protein family, a multigene calcium-binding family, comprises more than 20 members, each encoded by a separate gene. At least 16 of these genes cluster on chromosome 1q21 (6), known as the epidermal differentiation complex (7,9). In 1965, the first member of the $\mathrm{S} 100$ family was purified from bovine brain by Moore (10). Due to its solubility in a $100 \%$ saturated solution at neutral $\mathrm{pH}$ with ammonium sulfate $(11,12)$, it was termed the 'S100' protein. The $\mathrm{S} 100$ protein family is an acidic calcium-modulated protein family of low molecular weight (10-12 kDa), mainly expressed in vertebrates. It shares homology with calmodulin and other EF-hand type calcium-modulated proteins (11). Since then the expression of S100 proteins has been demonstrated in a diverse spectrum of tissues (5).

Structure of S100 proteins. S100 proteins belong to the calcium-binding EF-hand motif superfamily and have the 


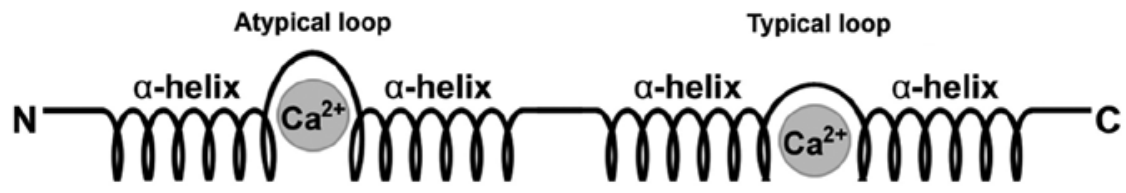

Figure 1. Schematic representation of the secondary structure of an S100 protein.

ability to form homodimers, heterodimers and oligomers (6). Each S100 protein is characterized by the presence of two $\mathrm{Ca}^{2+}$-modulated motifs of the EF-hand type interconnected by an intermediate region which is often referred to as the hinge region, resulting in a helix-loop-helix arrangement $(8,11,12)$ (Fig. 1).S100 proteins have two distinctEF-hands, one common to all EF-hand proteins on the $\mathrm{C}$-terminal portion and the other specific to the family located at the N-terminus. Subsequent to the C-terminal EF-hand region is a stretch of amino acids referred to as the $\mathrm{C}$-terminal extension. Between the two EF-hand domains is the area known as the hinge $(7,9)$. It is the $\mathrm{C}$-terminal extension and hinge areas that have the most variability among the different proteins and hence they are responsible for their specific biological properties (9).

Biological functions of S100 proteins. It is well documented that S100 proteins have a broad range of intracellular and extracellular functions (9). Intracellular functions include the regulation of enzyme activity and protein phosphorylation, calcium homeostasis, the regulation of cytoskeletal components and the regulation of transcriptional factors $(7,9,12)$ (Fig. 2).

Intracellular activities of $S 100$ proteins. S100 proteins play a wide range of roles in cells. The extracellular (9) and intracellular activities of S100 proteins include the regulation of adjusting key enzymes, calcium balance, the composition of the cytoskeleton, protein phosphorylation and dephosphorylation, as well as the regulation of energy metabolism $(13,14)$, cell differentiation and the cell cycle $(8,15)$. Members of the S100 family interact with p53 and these interactions with p53 produce differential effects, depending on the activity of the protein involved (9) (Fig. 3). Both S100A4 and S100B are thought to inhibit p53 phosphorylation, leading to the inhibition of its transcriptional activity, thereby compromising p53 tumor-suppressor activity (12). By contrast, S100A2 promotes p53 transcriptional activity (7). Thus the balanced actions of different S100 proteins within a cell determine its function. Many of the S100 family members are involved in modulating cytoskeletal dynamics. Again they display remarkable diversity of function, exhibiting direct interaction with tubulins, intermediate filaments, actin, myosin and tropomyosin. Some of these proteins have been implicated in mediating metastasis (8), such as S100A1 and S100A11. S100 protein members also play a role in regulating proliferation. In addition, most of the genes of the human S100 proteins are located on human chromosome lq21 $(7,9)$. Once a tumor develops, the genes in this area are easily reorganized and can interfere with the gene expression of S100 (9). Therefore, it is often observed that S100 proteins are accompanied by an abnormal expression in advanced cancer and metastasis. Thus, S100 proteins are closely related to the development and metastasis of a variety of tumors (9).

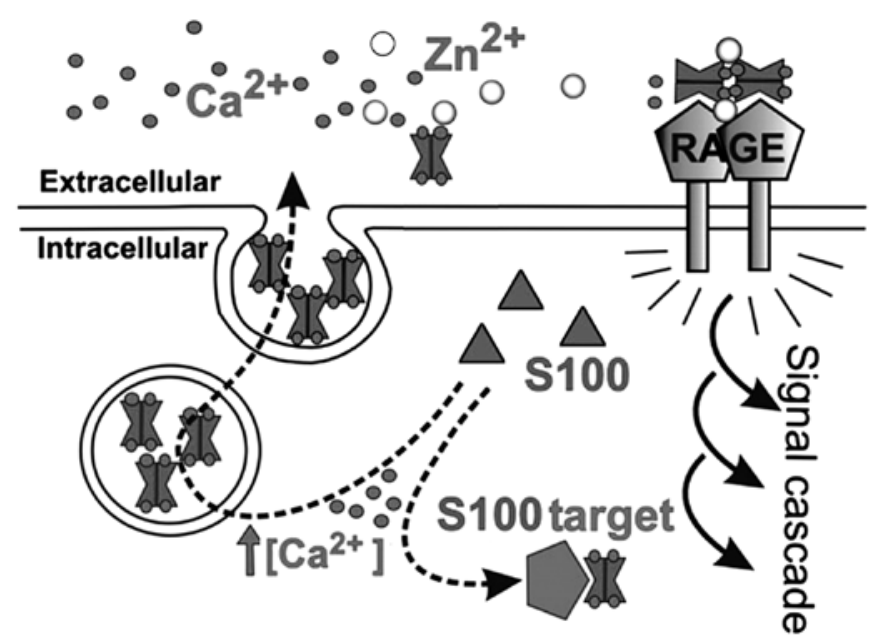

Figure 2. Intra- and extracellular functions of S100 proteins. S100 proteins act as $\mathrm{Ca}^{2+}$ sensor proteins in the cell and transmit a signal by $\mathrm{Ca}^{2+}$-dependent binding to a target protein, regulating its biological activity. Furthermore, several S100 proteins are secreted upon a $\mathrm{Ca}^{2+}$ signal. Extracellularly in the presence of high concentrations of $\mathrm{Ca}^{2+}$ and $\mathrm{Zn}^{2+}$, S100 proteins can form polymers and bind to the receptor, RAGE.

Extracellular roles of $S 100$ proteins. S100 proteins are involved in the extracellular stimulation of neuronal survival, differentiation and astrocyte proliferation, resulting in neuronal death via apoptosis, and stimulate (in some cases) or inhibit (in other cases) the activity of inflammatory cells $(9,16)$. S100 proteins are closely related to a variety of human diseases, such as neurological disorders, cancer, inflammation and heart disease (15).

\section{3. $\mathbf{S 1 0 0}$ proteins as molecular targets in pancreatic cancer}

S100 proteins interact with multiple molecular targets in pancreatic cancer. The key multiple molecular targets include the following:

Extracellular S100 proteins: interaction with receptor for advanced glycation end-products (RAGE). S100 proteins form heterodimers. These complexes display different affinities to target proteins, depending on their oligomerization state (17). This has been demonstrated for p53 and RAGE $(8,9,18)$. Structural analysis of receptor-ligand interaction has indicated that RAGE recognizes three-dimensional structures: one 'V-type' domain and two 'C-type' domains; a short transmembrane domain and a 43 -amino acid cytoplasmic tail $(19,20)$. The V-type domain has been found to confer ligand binding. The cytoplasmic tail is required for intracellular signaling, and the $\mathrm{V}$-type domain is responsible for ligand binding. Multiple 

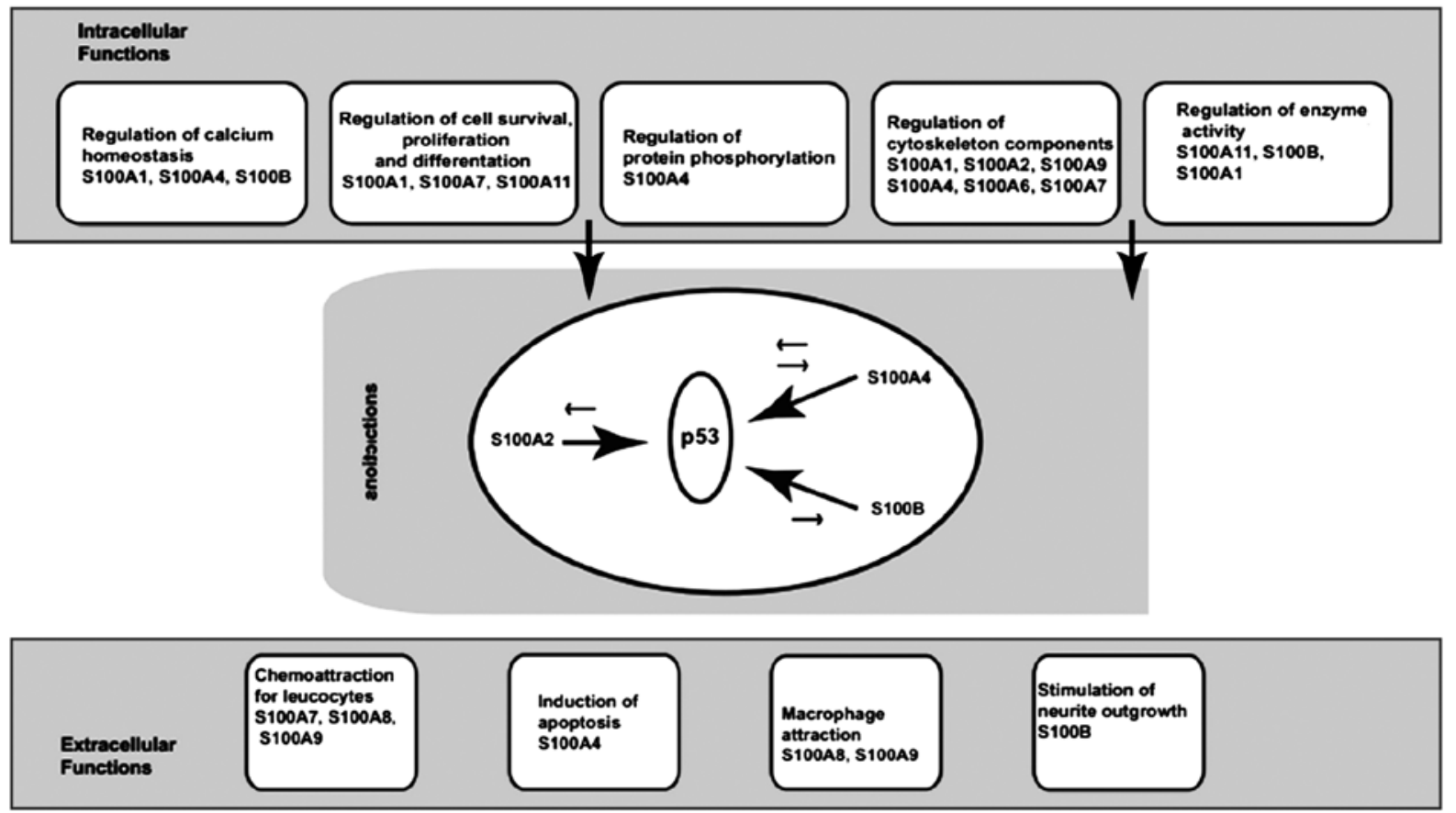

Figure 3. S100 proteins and their functions.

pathways downstream of RAGE have been identified, such as mitogen-activated protein (MAP) kinases, phosphatidylinositol 3-kinase (PI3K), Rho GTPases, nuclear factor (NF)- $\kappa \mathrm{B}$ and JAK/STAT $(8,21,22)$. RAGE has been shown to transduce the extracellular effects of S100B (22), S100A4, S100A6 (22), S100A8/A9 (23), S100A11 (24), S100A12, S100A13 and S100P $(19,20,25)$. The activation of RAGE by S100P stimulates cellular signaling pathways, including the MAP kinase and NF- $\kappa$ B pathways $(19,22,26)$. Certain studies have indicated that inhibiting S100P-RAGE interactions significantly reduces the basal levels of NF- $\kappa \mathrm{B}$ activity in pancreatic cancer and supports the existence of an autocrine loop involving RAGE ligands and RAGE in pancreatic cancer $(2,25,26)$. S100B and S100A6 have been shown not only to interact with distinct RAGE immunoglobulin domains, but also to exert opposite effects on cell survival $(27,28)$. At similar concentrations, S100B increases cellular proliferation (26), whereas S100A6 triggers apoptosis $(29,30)$. In addition, both S100 proteins induce the formation of reactive oxygen species (ROS); however, S100B recruits PI3K/AKT and NF- $\mathrm{BB}(31,32)$, whereas S100A6 activates JNK (30). The study by Arumugam et al also showed that S100A4 binding to RAGE was blocked by RAP (25). It has been reported that S100A4 may influence the resistance of pancreatic cancer cells to therapy $(16,33)$.

S100 proteins bind with cytoskeletal proteins and plasma membrane, thereby increasing cell migration. S100 proteins regulate all three major constituents of the cytoplasmic cytoskeleton, i.e. microtubules (MTs), intermediate filaments (IFs) and microfilaments (MFs) $(22,34)$, as well as tropomyosin and myosin $(8,35)$. S100 proteins can exert their effects depending on their interactions with cytoskeletal proteins and the membrane resulting in an enhancement of cell migration $(8,9)$.
For example, S100B and S100A1 disassemble cytoplasmic MTs and induce the aggregation of vimentin IFs as a result of in situ MT disassembly in triton-cytoskeletons from several cell lines (20). The consequent sequence potentially implicated in S100B binding has been identified in helix $\mathrm{H} 8$ of the central portion of tubulin and in its C-terminus, regions considered important for protofilament formation and, hence, for the MT assembly $(8,34,36)$. S100A4 interacts with cytoskeletal elements, such as actin, tubulin and non-muscle tropomyosin, establishing a direct role of S100A4 in regulating cell motility and cytoskeletal rearrangement (16). This suggests that S100A4 plays a possible mechanistic role in cell shape, motility and thus, invasion $(12,16)$. Other studies reported that S100A11 can associate with actin, $\beta$-tubulin, IFs and actin organization and Annexin I (37,38). S100A6 interacts with tropomyosin $\beta$, Annexin 11 and 2, and the novel binding protein, lamin B1, in pancreatic cancer cells. Hayes et al demonstrated that Annexin 2 was concentrated in the dynamic actin-rich protrusions of motile cells and that the siRNA-mediated depletion of Annexin 2 led to loss of protrusive and retractile activity (39). De Graauw et al pointed out that the phosphorylation of Annexin 2 was a key event in the remodelling of the actin cytoskeleton during cell spreading (40).

Interaction with p53. p53 is subjected to complex regulation. The biochemical activity of p53 as a transcription factor is adjusted by phosphorylation and acetylation, as well as modulation of protein stability, the degree of oligomerization, nuclear translocation and interactions with other components of the transcriptional machinery (12). The tumor suppressor protein, p53, plays a pivotal role in the maintenance and regulation of normal cellular functions through the induction of cell cycle arrest, DNA repair, or apoptosis in response to a 
variety of cellular stress signals and DNA damage $(8,12)$. In response to stress, p53 prevents tumorigenic transformation through the induction of cell cycle arrest or apoptosis $(22,41)$. p53 interacts with other components of the transcriptional machinery $(22,42)$. In unstressed cells, the expression level of p53 tumor suppressor is low $(43,44)$; however, upon stress challenge, p53 is activated by post-translational modifications that increase its stability $(45,46)$. The regulation of protein stability is one of the most effective mechanisms for controlling the function of p53 $(9,45)$. The key to this process is mouse double minute 2 (MDM2), an E3 ligase that targets p53 for ubiquitination. Several S100 proteins, such as S100B (46), S100A1, S100A2 (47), S100A4 (48), S100A6, S100A11 (24,32) and S100A14 (49) have been shown to interact with MDM2. Direct protein-protein interactions between S100 proteins and MDM2 promote the degradation of $\mathrm{p} 53$, as has been demonstrated for S100B and S100A4 $(12,48,50)$. This results in the loss of p53-dependent tumor suppressor activities. Elucidating the consequences of the metastasis-promoting activities of S100 proteins on p53-mediated functions, is of great importance in understanding cancer development and metastasis $(8,51)$. It is noteworthy that potential p53-binding sites have been identified in the promoter sequences of several S100 genes, indicating that the metastasis-promoting properties of S100 proteins are not as clear-cut as has been previously suggested. This is due to their interaction with p53-dependent apoptosis (22). This fact may explain why some members of the S100 family are markedly downregulated in malignant cells, in comparison to normal cells (22). Both S100A4 and S100B are thought to inhibit p53 phosphorylation, leading to the inhibition of its transcriptional activity, thereby compromising p53 tumor-suppressor activity $(16,41)$. By contrast, S100A2 promotes p53 transcriptional activity and of note, S100A4 has also been documented to enhance p53-dependent apoptosis $(16,47,50)$. Thus, the balance of actions of different S100 proteins within a cell can determine function (8).

Interaction with p21. p21/WAF1 is also known as cyclindependent kinase (CDK) inhibitor 1 or CDK-interacting protein 1. p21/WAF1 is a cell cycle checkpoint, where cells either set about repairing themselves or commit suicide through apoptosis $(52,53)$. The p21/WAF1 protein functions as a regulator of cell cycle progression at the G1 phase by inhibiting cyclin-CDK2 or -CDK1 activity $(52,54,55)$. The expression of its gene is tightly controlled by $\mathrm{p} 53$, through which the p21/WAF1 protein mediates p53-dependent cell cycle G1 phase arrest, in response to a variety of stress stimuli $(24,55)$. For example, S100A11 has been shown to be involved since TGF- $\beta$ induces S100A11 gene expression and translocation into the nucleus, where it interacts with p21/WaF1 $(56,57)$. S100A4 target genes comprise p21/WAF, Bax, thrombospondin-1 and MDM2 $(16,58,59)$.

S100 proteins: role in the degradation of the extracellular matrix (ECM) and metastasis. Angiogenesis is a crucial step in cancer progression, as it supplies the proliferating tumor cells with necessary nutrients and oxygen and at the same time, it provides an escape route for invading tumor cells (60). Matrix metalloproteinases (MMPs) promote metastasis both by degrading the ECM and promoting and maintaining angio- genic characteristics (16). An important factor affecting the motility of cancer cells is the degradation of the ECM (22). S100 proteins have a variety of molecular mechanisms. For example, the metastatic function of S100A4 is associated with its ability to upregulate the expression of several MMPs. S100A4 gene suppression significantly decreases the expression of MMP-9, while the overexpression of the S100A4 gene significantly increases MMP-9 expression $(12,16,18)$. Extracellular S100A4 binds to RAGE, and upregulates MMP-13, MMP-2 and MMP-9 gene expression $(19,61)$, allowing cell invasion and thus promoting metastasis $(1,16)$. Intracellular S100A14 promotes cell motility and invasiveness by regulating the expression and function of MMP-2 in a p53-dependent manner $(22,62)$. p53 transrepresses MMP-2 gene expression and thus enables intracellular S100A14 to effect p53 transactivity and stability, resulting in an enhancement of MMP2 gene expression (22). S100A8/A9 overexpression can also induce the upregulation of MMP-9 in HaCaT keratinocytes. MMP9 gene induction depends on NF- $\kappa \mathrm{B}$ activation and intracellular S100A8/A9 has been shown to promote epithelial NADPH oxidases and subsequently, NF- $\kappa \mathrm{B}$ activation (63). S100P induces the expression of cathepsin D, an aspartyl protease, which takes part in the proteolytic degradation of the ECM. Hence it increases the invasive potential of the tumor $(2,22)$.

\section{4. $\mathrm{S100}$ protein expression in pancreatic cancer}

Multiple proteins of the $\mathrm{S} 100$ protein family are closely related to pancreatic cancer, including the following proteins:

S100A2. The S100A2 gene is located on the long arm of Area 2 of chromosome 1 . The chromosomal stability of this section is poor, and closely related to tumor development $(47,64)$. S100A2 is often expressed in normal cells and regulated by cell cycle progression and the tumor suppressor gene, p53 (47). The lack of S100A2 has been proven to be associated with the development of numerous human tumors. Since it is absent in the majority of tumor types, S100A2 is very important in normal tissue growth and differentiation (65). Studies have demonstrated that the lack of S100A2 functionality may be due to the selective hypermethylation in the promoter region. In tumor cells, a transcription factor binds to S100A2 promoter, which leads to hypermethylation and, consequently, to the transcriptional silencing of S100A2, thereby rendering it non-reactive with most tumor suppressor genes (65). Ohuchida et al microdissected invasive ductal carcinoma (IDC), pancreatic intraepithelial neoplasia (PanIN), intraductal papillary mucinous neoplasm (IPMN), pancreatitis-affected epithelial (PAE) and normal ductal cells and then studied S100A2 expression by quantitative reverse transcription PCR (qRT-PCR) (27). The analyses revealed that IDC cells expressed higher levels of S100A2 than did IPMN, PAE or normal cells (27). Cell lines from metastatic sites expressed higher levels of S100A2 than those from primary sites. PanIN cells expressed higher levels of S100A2 than normal cells. IDC cells associated with poorly differentiated adenocarcinoma expressed higher levels of S100A2 than did IDC cells without poorly differentiated adenocarcinoma (27). Analyses of formalin-fixed paraffin-embedded (FFPE) samples revealed that the expression levels of S100A2 were higher in samples from patients who survived $<1,000$ days after surgery 
than in those from patients who survived $>1,000$ days (27). S100A2 may be a marker of tumor progression or prognosis in pancreatic carcinogenesis and pancreatic cancer $(65,66)$. Moreover, S100A 2 has recently been suggested to be a negative prognostic biomarker in pancreatic cancer. Biankin et al (67) demonstrated that patients with S100A2-negative tumors had a significant survival benefit from pancreatectomy even in the presence of involved surgical margins or lymph node metastasis. S100A2 expression is a good predictor of the response to pancreatectomy for pancreatic cancer. Data suggest that a high S100A2 expression may be a marker of a metastatic phenotype $(67,68)$. The prospective measurement of S100A2 expression in diagnostic biopsy samples has potential clinical utility as a predictive biomarker of response to pancreatectomy and other therapies that target locoregional disease $(67,68)$. These data demonstrate that S100A2 is associated with tumor progression in pancreatic cancer and is a negative prognostic biomarker in pancreatic cancer.

S100A4. The S100A4 protein consists of 101 amino acids, it is an $11-\mathrm{kDa}$ molecular weight protein and exists as noncovalent dimers in the cell and as covalent dimers in the extracellular domain $(16,69)$. In normal lung, kidney, breast, thyroid, pancreas and colon tissue cells, the expression of the S100A4 protein is absent. Studies have shown that the expression of S100A4 protein in pancreatic cancer is significantly higher than in adjacent normal pancreatic tissue, and that the expression of S100A4 in poorly differentiated pancreatic tissue and metastatic pancreatic cancer tissue (66) is significantly higher than that in well-differentiated and non-metastatic pancreatic cancer tissue $(16,70)$. The overexpression of S100A4 protein is associated with hypomethylation of the first intron of the corresponding gene, leading to poor differentiation of pancreatic cancer (71). S100A4 protein overexpression also plays an important role in pancreatic cancer invasion and in the metastasis process (71). Another study demonstrated that S100A4-silenced cells exhibited a marked decrease in migration and invasiveness and increased adhesion, whereas overall proliferation and apoptosis were not overtly altered (1). S100A4 and its downstream factors play important roles in pancreatic cancer invasion. A100A4 silencing can significantly restrain the invasiveness of pancreatic cancer (1). Studies have suggested that the S100A4 protein is an independent prognostic factor of pancreatic cancer which can differentiate pancreatic cancer from lymph node metastasis $(68,72,73)$.

S100A6. The S100A6 gene is a single-copy gene, located on human chromosome lq21, adjacent to ski proto-oncogene (74). S100A6 consists of 90 amino acids. The S100A6 protein may be relevant to pancreatic cancer prognosis (30). Ohuchida et al $(75,76)$ analyzed the secretion of S100A6 protein expression in normal pancreatic tissue, PanIN and IDC of the pancreas, and found that the level of S100A6 expression in pancreatic cancer was significantly higher than that in noncancerous tissue. S100A6 protein expression in cancerous pancreatic juice was significantly higher than that in normal pancreatic juice (35). Vimalachandran et al (77) showed that the expression of S100A6 in pancreatic cancer cell nuclei was significantly higher compared with the cytoplasm. Patients with high S100A6 protein expression levels in the nucleus presented with poor prognosis (77). However, the expression level of cytoplasmic S100A6 had no clear association with prognosis. An absence of S100A6 expression was observed during PanIN period. However, as PanIN levels increased, S100A6 protein expression levels gradually increased as well, particularly in the nucleus (77). This indicated that even though S100A6 protein expression in pancreatic cancer is an early event, it is the expression of S100A6 in the nucleus that can be used as an independent prognostic factor. Its high expression levels often precede a poor prognosis. However, there is no evidence to date to support a correlation between S100A6 and the occurrence, differentiation and metastasis of pancreatic cancer $(75,76,77)$.

S100P. S100P is a 95-amino-acid protein whose gene is located on chromosome 4 pl6 (42). S100P protein monomers have shown a positive correlation with calcium-dependent binding; whereas the dissociation rate constant was independent of calcium (2). The dimer contact surface and the core area of the hydrophobic amino acid mutation S100P proteins affect polymerization. S100P and its ligand, S100P BPR, coexist in the nucleus. In situ hybridization has confirmed the presence of the S100P BPR transcription product in normal pancreatic islet cells and pancreatic ductal adenocarcinoma, which was not expressed in normal pancreatic duct cells $(78,79)$. As shown by qRT-PCR, S100P and S100PBPR were observed in PanIN and pancreatic cancer tissue specimens. These results suggest that S100P and S100PBPR play a role in early pancreatic cancer occurrence $(78,79)$. The expression level of S100P is positively associated with PanIN. The gradual increase in S100P concentration is expressed as PanlN-1, PanIN-2 and PanIN-3. This indicates that S100P plays an important role in the progression from PanIN to invasive ductal adenocarcinoma in the pancreas $(78,79)$. Ohuchida et al $(80)$, as well as others examined the expression levels of S100P in various other pancreatic diseases. According to organizational analyses it was found that pancreatic cancer and IPMN tissue expressed significantly higher levels of S100P than did tissue from nonneoplastic pancreas $(80,81)$. Microdissection analyses revealed that IPMN tissue expressed significantly higher levels of S100P than did pancreatic cancer and PanIN tissue $(80,81)$. There was no significant difference between the expression levels of pancreatic cancer and PanIN. In pancreatic juice analyses, S100P expression levels in patients with pancreatic cancer and IPMN were significantly higher than those in patients with pancreatitis $(80,81)$. Thus, neoplastic disease can be effectively distinguished from chronic pancreatitis. S100P has been shown to mediate tumor growth, drug resistance and metastasis through RAGE $(26,42,80)$. Arumugam et al (90) demonstrated that elevated expression levels of S100P in mice accelerated the growth rate of pancreatic cancer cells, contrary to its decreased expression levels which delayed cancer cell growth. These studies demonstrate that the S100P protein plays an important role in the incidence of pancreatic cancer. S100P favors the early diagnosis of pancreatic cancer (82). It can be used as an early diagnostic biomarker for pancreatic cancer by detecting its expression levels in the pancreatic juice of the patient. The expression of S100P has been shown to be associated with drug resistance, metastasis and poor clinical outcome $(2,83)$. 
S100A11. S100A11 also known as S100C (55), is a member of the family of S100 proteins, and was first discovered in 1989 (55). The S100A11 protein consists of 99 amino acids, with a molecular weight of $11 \mathrm{kDa}$. Its gene is located on chromosome 1q21 $(55,56)$. The detection of S100A11 RNA expression levels in different tissues has indicated that the highest expression levels are present in the placenta, heart, kidneys and lungs, whereas moderate expression levels are present in skeletal muscle tissues and the lowest in the brain tissue $(55,84)$. Higher levels of S100A11 protein are expressed in duct cells of different tissues, while lower levels are detected in the epithelial cells of the digestive tract. The S100A11 protein is mainly distributed in the nucleus, and the remaining S100A11 protein is distributed in the cytoplasm $(55,85)$. Sakaguchi et al (53) reported that S100A11 increases the transcription of p21, a negative regulator of cell growth $(66,85)$. In addition, the expression of several known tumor suppressor genes, including p53 and p16INK4 (86), has been reported to be increased in premalignant lesions; S100A11 expression is elevated in noninvasive neoplasms, such as IPMA and PanIN, but decreased in invasive cancer, such as IDC $(87,88)$. The expression of S100A11 protein has been shown to be significantly decreased within human fibroblasts that have undergone malignant transformation (57). The enforced expression of S100A11 protein has been shown to significantly inhibit the growth of malignant cells. Data suggest that S100A11 may be a tumor suppressor gene (56). Ohuchida et al also showed that S100A11 expression increased during the early stages of pancreatic cancer and then decreased as cancer progressed (57). Previous studies have also shown that S100A11 functions as a dual cell growth mediator as it is highly expressed in pancreatic tissue, and its high expression in pancreatic cancer is associated with tumor differentiation and lymph node metastasis $(56,84)$. S100A11 is a significant tumor marker for pancreatic cancer and an unfavorable predictor for the prognosis of patients who have undergone surgical resection $(84,89)$. We consider S100A11 relevant with the occurrence and development of pancreatic cancer. In conclusion, S100A11 is a putative tumor suppressor gene. It can be used as an independent prognostic indicator of pancreatic cancer, particularly for the early diagnosis of pancreatic cancer. S100A11 analysis in pancreatic juice may allow the early detection of pancreatic cancer and the effective screening of patients with high-risk lesions that may progress to pancreatic cancer, such as patients who have a family history of pancreatic cancer or who have chronic pancreatitis (57).

\section{Conclusion}

S100 proteins interact with RAGE, p53 and p21, play a role in the degradation of the ECM and metastasis, and bind with cytoskeletal proteins and the plasma membrane in pancreatic cancer progression and metastasis. S100A11 and S100P are significant tumor markers for pancreatic cancer and unfavorable predictors for the prognosis of patients who have undergone surgical resection. S100A2 has recently been suggested to be a negative prognostic biomarker in pancreatic cancer. The expression of S100A6 in the nucleus may used as an independent prognostic pancreatic factor. The expression of S100A4 and S100P is associated with drug resistance, differentiation, metastasis and clinical outcome. The data presented in this review suggest that $\mathrm{S} 100$ proteins may be used as molecular markers for the early diagnosis, treatment and prognosis of pancreatic cancer.

\section{Acknowledgements}

This study was supported by grants from the Foundation for Talents in Six Fields of Jiangsu Province (no. 2012-WSN-065), the Health Project of Jiangsu Province (no. H201318) and the Social Development Foundation of Nantong City (nos. S2010012, HS2011004 and BK2013069).

\section{References}

1. Li N, Song MM, Chen XH, Liu LH and Li FS: S100A4 siRNA inhibits human pancreatic cancer cell invasion in vitro. Biomed Environ Sci 25: 465-470, 2012.

2. Arumugam T and Logsdon CD: S100P: a novel therapeutic target for cancer. Amino acids 41: 893-899, 2011.

3. Xie L, Ni WK, Chen XD, Xiao MB, Chen BY, He S, Lu CH, Li XY, Jiang F and Ni RZ: The expressions and clinical significances of tissue and serum galectin-3 in pancreatic carcinoma. J Cancer Res Clin Oncol 138: 1035-1043, 2012.

4. Danovi SA, Wong HH and Lemoine NR: Targeted therapies for pancreatic cancer. Br Med Bull 87: 97-130, 2008

5. Rezvanpour A and Shaw GS: Unique S100 target protein interactions. Gen Physiol Biophys 28: F39-F46, 2009.

6. Yao R, Lopez-Beltran A, Maclennan GT, Montironi R, Eble JN and Cheng L: Expression of S100 protein family members in the pathogenesis of bladder tumors. Anticancer Res 27: 3051-3058, 2007.

7. Salama I, Malone PS, Mihaimeed F and Jones JL: A review of the S100 proteins in cancer. Eur J Surg Oncol 34: 357-364, 2008.

8. Donato R: S100: a multigenic family of calcium-modulated proteins of the EF-hand type with intracellular and extracellular functional roles. Int J Biochem Cell Biol 33: 637-668, 2001.

9. Marenholz I, Heizmann CW and Fritz G: S100 proteins in mouse and man: from evolution to function and pathology (including an update of the nomenclature). Biochem Biophys Res Commun 322: 1111-1122, 2004.

10. Moore BW: A soluble protein characteristic of the nervous system. Biochem Biophys Res Commun 19: 739-744, 1965.

11. Zimmer DB, Cornwall EH, Landar A and Song W: The S100 protein family: history, function, and expression. Brain Res Bull 37: 417-429, 1995.

12. Berge G and Mælandsmo GM: Evaluation of potential interactions between the metastasis-associated protein S100A4 and the tumor suppressor protein p53. Amino Acids 41: 863-873, 2011.

13. Komatsu K, Kobune-Fujiwara Y, Andoh A, et al: Increased expression of S100A6 at the invading fronts of the primary lesion and liver metastasis in patients with colorectal adenocarcinoma. Br J Cancer 83:769-774, 2000.

14. Melle C, Ernst G, Schimmel B, Bleul A and Eggeling FV: Colonderived liver metastasis, colorectal carcinoma, and hepatocellular carcinoma can be discriminated by the $\mathrm{Ca}\left({ }^{2+}\right)$-binding proteins S100A6 and S100A11. PloS One 3: e3767, 2008.

15. Schäfer BW and Heizmann CW: The S100 family of EF-hand calcium-binding proteins: functions and pathology. Trends Biochem Sci 21: 134-140, 1996.

16. Mishra SK, Siddique HR and Saleem M: S100A4 calcium-binding protein is key player in tumor progression and metastasis: preclinical and clinical evidence. Cancer Metastasis Rev 31: 163-172, 2012.

17. Heizmann CW, Ackermann GE and Galichet A: Pathologies involving the S100 proteins and RAGE. Subcell Biochem 45: 93-138, 2007.

18. Saleem M, Kweon MH, Johnson JJ, et al: S100A4 accelerates tumorigenesis and invasion of human prostate cancer through the transcriptional regulation of matrix metalloproteinase 9. Proc Natl Acad Sci USA 103: 14825-14830, 2006.

19. Gross SR, Sin CG, Barraclough R and Rudland PS: Joining S100 proteins and migration: for better or for worse, in sickness and in health. Cell Mol Life Sci 30: June 30, 2013 (Epub ahead of print). 
20. Sorci G, Riuzzi F, Giambanco I and Donato R: RAGE in tissue homeostasis, repair and regeneration. Biochim Biophys Acta 1833: 101-109, 2012.

21. Xie J, Méndez JD, Méndez-Valenzuela and AguilarHernández MM: Cellular signalling of the receptor for advanced glycation end products (RAGE). Cell Signal 25: 2185-2197, 2013.

22. Maletzki C, Bodammer P, Breitrück A and Kerkhoff C: S100 proteins as diagnostic and prognostic markers in colorectal and hepatocellular carcinoma. Hepat Mon 12: e7240, 2012.

23. Volz HC, Laohachewin D, Seidel C, et al: S100A8/A9 aggravates post-ischemic heart failure through activation of RAGE-dependent NF- $\kappa$ B signaling. Basic Res Cardiol 107: 250, 2012.

24. Hung KW, Chang YM and Yu C: Resonance assignments of $\mathrm{Ca}^{2+}$-bound human S100A11. Biomol NMR Assign 7: 211-214, 2013.

25. Arumugam T, Ramachandran V, Gomez SB, Schmidt AM and Logsdon CD: S100P-derived RAGE antagonistic peptide reduces tumor growth and metastasis. Clin Cancer Res 18: 4356-4364, 2012.

26. Arumugam T, Ramachandran V, Sun D, et al: Designing and developing S100P inhibitor 5-methyl cromolyn for pancreatic cancer therapy. Mol Cancer Ther 12: 654-662, 2013.

27. Ohuchida K, Mizumoto K, Miyasaka Y, et al: Over-expression of S100A2 in pancreatic cancer correlates with progression and poor prognosis. J Pathol 213: 275-282, 2007.

28. Leclerc E, Fritz G, Weibel M, Heizmann CW and Calichet A: S100B and S100A6 differentially modulate cell survival by interacting with distinct RAGE (receptor for advanced glycation end products) immunoglobulin domains. J Biol Chem 282: 31317-31331, 2007

29. Leclerc E and Heizmann CW: The importance of $\mathrm{Ca}^{2+} / \mathrm{Zn}^{2+}$ signaling S100 proteins and RAGE in translational medicine. Front Biosci (Schol Ed) 3: 1232-1262, 2011.

30. Filipek A, Michowski W and Kuznicki J: Involvement of S100A6 (calcyclin) and its binding partners in intracellular signaling pathways. Adv Enzyme Regul 48: 225-239, 2008.

31. Huttunen HJ, Kuja-Panula J, Sorci G, Agneletti AL, Donato R and Rauuala H: Coregulation of neurite outgrowth and cell survival by amphoterin and S100 proteins through receptor for advanced glycation end products (RAGE) activation. J Biol Chem 275: 40096-40105, 2000

32. Taguchi A, Blood DC, del Toro G, et al: Blockade of RAGE-amphoterin signalling suppresses tumour growth and metastases. Nature 405: 354-360, 2000.

33. Lukanidin E and Sleeman JP: Building the niche: the role of the S100 proteins in metastatic growth. Semin Cancer Biol 22: 216-225, 2012

34. Chen H, Fernig DG, Rudland PS, Sparks A, Wilkinson MC and Barraclough R: Binding to intracellular targets of the metastasis-inducing protein, S100A4 (p9Ka). Biochem Biophys Res Commun 286: 1212-1217, 2001.

35. Kriajevska MV,Cardenas MN, Grigorian MS, Ambartsumian NS Georgiev GP and Lukanidin EM: Non-muscle myosin heavy chain as a possible target for protein encoded by metastasis-related mts-1 gene. J Biol Chem 269: 19679-19682, 1994.

36. Zimmer DB and Van Eldik LJ: Analysis of the calciummodulated proteins, S100 and calmodulin, and their target proteins during C6 glioma cell differentiation. J Cell Biol 108: 141-151, 1989.

37. Zhao XQ, Naka M, Muneyuki M and Tanaka T: $\mathrm{Ca}(2+)$-dependent inhibition of actin-activated myosin ATPase activity by S100C (S100A11), a novel member of the S100 protein family. Biochem Biophys Res Commun 267: 77-79, 2000.

38. Broome AM and Eckert RL: Microtubule-dependent redistribution of a cytoplasmic cornified envelope precursor. J Invest Dermatol 122: 29-38, 2004

39. Hayes MJ, Shao D, Bailly M and Moss SE: Regulation of actin dynamics by annexin 2. EMBO J 25: 1816-1826, 2006.

40. de Graauw M, Tijdens I, Smeets MB et al: Annexin A2 phosphorylation mediates cell scattering and branching morphogenesis via cofilin activation. Mol Cell Biol 28: 1029-1040, 2008.

41. Rust RR, Baldisseri DM and Weber DJ: Structure of the negative regulatory domain of p53 bound to S100B (betabeta). Nat Struct Biol 7: 570-574, 2000

42. Kato K, Kamada H, Fujimori T, Aritomo Y, Ono M and Masaki T: Molecular biologic approach to the diagnosis of pancreatic carcinoma using specimens obtained by EUS-guided fine needle aspiration. Gastroenterol Res Pract 2012: 243524, 2012
43. Sablina AA, Budanov AV, Ilyinskaya GV, Agapova LS, Kravchenko JE and Chumakov PM: The antioxidant function of the p53 tumor suppressor. Nat Med 11: 1306-1313, 2005.

44. Green ML, Pisano MM, Prough RA and Knudsen TB: Release of targeted p53 from the mitochondrion as an early signal during mitochondrial dysfunction. Cell Signal 25: 2383-2390, 2013.

45. Leśniak W, Słomnicki LP and Filipek A: S100A6-new facts and features. Biochem Biophys Res Commun 390: 1087-1092, 2009.

46. Van Dieck J, Lum J K, Teufel D P and Fersht AR: S100 proteins interact with the N-terminal domain of MDM2. FEBS Letts 584 3269-3274, 2010 .

47. Wolf S, Haase-Kohn C and Pietzsch J: S100A2 in cancerogenesis: a friend or a foe? Amino Acids 41: 849-861, 2011.

48. Nishioku T, Furusho K, Tomita A, et al: Potential role for S100A4 in the disruption of the blood-brain barrier in collagen-induced arthritic mice, an animal model of rheumatoid arthritis. Neuroscience 189: 286-292, 2011.

49. Sapkota D, Costea DE, Blø M, Bruland O, Lorens JB, Vasstrand EN and Ibrahim SO: S100A14 inhibits proliferation of oral carcinoma derived cells through G1-arrest. Oral Oncol 48: 219-225, 2012.

50. Grigorian M, Andresen S, Tulchinsky E, et al: Tumor suppressor p53 protein is a new target for the metastasis-associated Mts1/S100A4 protein: functional consequences of their interaction. J Biol Chem 276: 22699-22708, 2001.

51. Shimamoto S, Kubota Y, Yamaguchi F, Tokumitsu H and Kobayashi R: $\mathrm{Ca}^{2+} / \mathrm{S} 100$ proteins act as upstream regulators of the chaperone-associated ubiquitin ligase $\mathrm{CHIP}$ (C terminus of Hsc70-interacting protein). J Biol Chem 288: 7158-7168, 2013.

52. Romanov VS, Pospelov VA and Pospelova TV: Cyclin-dependent kinase inhibitor p21(Waf1): contemporary view on its role in senescence and oncogenesis. Biochemistry (Mosc.) 77: 575-584, 2012.

53. Sakaguchi M, Miyazaki M, Takaishi M, et al: S100C/A11 is a key mediator of $\mathrm{Ca}\left({ }^{2+}\right)$-induced growth inhibition of human epidermal keratinocytes. J Cell Biol 163: 825-835, 2003.

54. Li B, Wan X, Zhu Q, et al: Net expression inhibits the growth of pancreatic ductal adenocarcinoma cell PL45 in vitro and in vivo. PloS One 8: e57818, 2013.

55. Sakaguchi M and Huh NH: S100A11, a dual growth regulator of epidermal keratinocytes. Amino Acids 41: 797-807, 2011.

56. He H, Li J, Weng S, LI M and Yu Y: S100A11: diverse function and pathology corresponding to different target proteins. Cell Biochem Biophys 55: 117-126, 2009.

57. Ohuchida K, Mizumoto K, Ohhashi S, et al: S100A11, a putative tumor suppressor gene, is overexpressed in pancreatic carcinogenesis. Clin Cancer Res 12: 5417-5422, 2006.

58. Mann K and Hainaut P: Aminothiol WR1065 induces differential gene expression in the presence of wild-type p53. Oncogene 24: 3964-3975, 2005.

59. Brain JG, Robertson H, Thompson E, et al: Biliary epithelial senescence and plasticity in acute cellular rejection. Am J Transplant 13:1688-1702, 2013.

60. Yang SY, Miah A, Pabari A and Winslet M: Growth Factors and their receptors in cancer metastases. Front Biosci (Landmark Ed) 16: 531-538, 2011

61. Jia W, Gao XJ, Yang ZX and Zhang ZD: S100A4 silencing suppresses proliferation, angiogenesis and invasion of thyroid cancer cells through downregulation of MMP-9 and VEGF. Eur Rev Med Pharmacol Sci 17: 1495-1508, 2013.

62. Chen H, Yuan Y, Zhang C, et al: Involvement of S100A14 protein in cell invasion by affecting expression and function of matrix metalloproteinase (MMP)-2 via p53-dependent transcriptional regulation. J Biol Chem 287: 17109-17119, 2012.

63. Gebhardt C, Németh J, Angel P and Hess J: S100A8 and S100A9 in inflammation and cancer. Biochem Pharmacol 72: 1622-1631, 2006.

64. Hsu TC, Young MR, Cmarik J and Colburn NH: Activator 1 (AP-1)- and nuclear factor kappaB (NF-kappaB)-dependent transcriptional events in carcinogenesis. Free Radic Biol Med 28: $1338-48,2000$.

65. Bachet JB, Maréchal R, Demetter P, et al: S100A2 is a predictive biomarker of adjuvant therapy benefit in pancreatic adenocarcinoma. Eur J Cancer 49: 2643-53, 2013.

66. Jamieson NB, Carter CR, McKay CJ and Oien KA: Tissue biomarkers for prognosis in pancreatic ductal adenocarcinoma: a systematic review and meta-analysis. Clin Cancer Res 17: 3316-3331, 2011. 
67. Biankin AV, Kench JG, Colvin EK, et al: Expression of S100A2 calcium-binding protein predicts response to pancreatectomy for pancreatic cancer. Gastroenterology 137: 558-568, 2009.

68. Russo SM, Ove R and Saif MW: Identification of prognostic and predictive markers in pancreatic adenocarcinoma. Highlights from the '2011 ASCO Gastrointestinal Cancers Symposium'. San Francisco, CA, USA. January 20-22, 2011. JOP 12: 92-95, 2011

69. Sekine H, Chen N, Sato K, et al: S100A4, frequently overexpressed in various human cancers, accelerates cell motility in pancreatic cancer cells. Biochem Biophys Res Commun 429: 214-219, 2012.

70. Ilg EC, Schäfer BW and Heizmann CW: Expression pattern of S100 calcium-binding proteins in human tumors. Int J Cancer 68: 325-332, 1996.

71. Tsukamoto N, Egawa S, Akada M, et al: The expression of S100A4 in human pancreatic cancer is associated with invasion. Pancreas 42: 1027-1033, 2013.

72. Chang D, Colvin E, Scarlett C, et al: A molecular prognostic nomogram for resectable pancreatic cancer. J Clin Oncol 29 (Suppl 4): abs. 154, 2011.

73. Ikenaga N, Ohuchida K, Mizumoto K, et al: S100A4 mRNA is a diagnostic and prognostic marker in pancreatic carcinoma. J Gastrointest Surg 13: 1852-1858, 2009.

74. Schneider G and Filipek A: S100A6 binding protein and Siah-1 interacting protein (CacyBP/SIP): spotlight on properties and cellular function. Amino Acids 41: 773-780, 2011.

75. Ohuchida K, Mizumoto K, Ishikawa N, et al: The role of S100A6 in pancreatic cancer development and its clinical implication as a diagnostic marker and therapeutic target. Clin Cancer Res 11: 7785-7793, 2005.

76. Ohuchida K, Mizumoto K, Yu J, et al: S100A6 is increased in a stepwise manner during pancreatic carcinogenesis: clinical value of expression analysis in 98 pancreatic juice samples. Cancer Epidemiol Biomarkers Prev 16: 649-654, 2007.

77. Vimalachandran D, Greenhalf W, Thompson C, et al: High nuclear S100A6 (Calcyclin) is significantly associated with poor survival in pancreatic cancer patients. Cancer Res 65: 3218-3225, 2005.

78. Dowen SE, Crnogorac-Jurcevic T, Gangeswaran R, et al Expression of S100P and its novel binding partner S100PBPR in early pancreatic cancer. Am J Pathol 166: 81-92, 2005.

79. Han H, Bearss DJ, Browne LW, Calaluce R, Nagle RB and Von Hoff DD: Identification of differentially expressed genes in pancreatic cancer cells using cDNA microarray. Cancer Res 62: 2890-2896, 2002 .
80. Ohuchida K, Mizumoto K, Egami T, et al: S100P is an early developmental marker of pancreatic carcinogenesis. Clin Cancer Res 12: 5411-5416, 2006.

81. Nakata K, Nagai E, Ohuchida K, et al: $\mathrm{S} 100 \mathrm{P}$ is a novel marker to identify intraductal papillary mucinous neoplasms. Hum Pathol 41: 824-831, 2010.

82. Crnogorac-Jurcevic T, Missiaglia E, Blaveri E, et al: Molecular alterations in pancreatic carcinoma: expression profiling shows that dysregulated expression of S100 genes is highly prevalent J Pathol. 201: 63-74, 2003.

83. Barry S, Chelala C, Lines K, et al: S100P is a metastasisassociated gene that facilitates transendothelial migration of pancreatic cancer cell. Clin Exp Metastasis 30: 251-264, 2013.

84. Inada $H$, Naka M, Tanaka T, Davey GE and Heizmann CW: Human S100A11 exhibits differential steady-state RNA levels in various tissues and a distinct subcellular localization. Biochem Biophys Res Commun 263: 135-138, 1999.

85. Chen JH, Ni RZ, Xiao MB, Guo JG and Zhou JW: Comparative proteomic analysis of differentially expressed proteins in human pancreatic cancer tissue. Hepatobiliary Pancreat Dis Int 8: 193-200, 2009

86. Memon AA, Sorensen BS, Meldgaard P, Fokdal L, Thykjaer T and Nexo E: Down-regulation of S100C is associated with bladder cancer progression and poor survival. Clin Cancer Res 11: 606-611, 2005.

87. Nakashima T, Wang XF, Masuda M, Inokuchi A and Komiyama S: Overexpression of $\mathrm{p} 53$ nuclear protein in premalignant and malignant laryngeal lesions. Eur Arch Otorhinolaryngol 256: S56-S59, 1999.

88. Shin DM, Kim J, Ro JY, Hittelman J, Roth JA, Hong WK and Hittelman WN: Activation of p53 gene expression in premalignant lesions during head and neck tumorigenesis. Cancer Res 54: 321-326, 1994.

89. Xiao MB, Jiang F, Ni WK, Chen BY, Lu CH, Li XY and Ni RZ: High expression of S100A11 in pancreatic adenocarcinoma is an unfavorable prognostic marker. Med Oncol 29: 1886-1891, 2012.

90. Arumugam T, Simeone DM, Van Golen K and Logsdon CD: S100P promotes pancreatic cancer growth, survival, and invasion. Clin Cancer Res 11: 5356-5364, 2005. 